\title{
Selection Method Study on the Best Grounding Resistance for the Neutral Point of 10kV Urban Power Distribution System
}

\author{
Shengsuo Niu ${ }^{a}$, Hui $\mathrm{Wu}^{\mathrm{b}}$ and Zhirui Liang ${ }^{\mathrm{c}}$ \\ School of North China Electric Power University, Baoding 071003, China. \\ aniushengsuo@163.com, b404887097@qq.com, cZhiruil@sohu.com
}

\begin{abstract}
With the rapid growth of urban load and the increase of cable rates in urban distribution network, the shortcomings of traditional neutral point grounding are more and more obvious, which lead to the application of resistance grounding. Considering the technical and economic factors, this paper presents a method for rational selection of neutral grounding resistance. In this method, on one hand, it uses technical requirements as constraints to limit the range of grounding resistance, on the other hand, it uses optimal economy as the objective function to choose the best grounding resistance. Furthermore, the result of example verifies the feasibility and effectiveness of the method.
\end{abstract}

Keywords: Urban distribution network; neutral grounding modes; resistance grounding; grounding resistance value.

\section{Introduction}

The current literature simply refers to select the resistance value according to the size of fault current of neutral point grounding, for the selection of neutral resistance value in $10-35 \mathrm{kv}$ distribution network. Based on the factors which influence the selection of neutral grounding resistance, this paper puts forward the optimal selection method, studying both economy and technicality factors.

\section{The optimal selection method of neutral grounding resistance}

In this paper, it uses technical requirements as constrains and optimal economy as the objective function to establish the optimal selection model of neutral grounding resistance.

\subsection{Determination of constraint}

The constraints are requirements to neutral grounding resistance from four aspects: internal overvoltage in system, relay protection, interference to telecommunication lines and personal safety. When metallic one-phase ground fault occurs in the resistance grounding system, a simplified equivalent circuit has appeared as Figure 1 shows.

The neutral point resistance from Figure 1: $\dot{U}_{0}=-\hat{E}_{A}$; fault current caused by short circuit:

$\dot{I}_{K}=\dot{U}_{0}\left(\frac{1}{R_{N}}+j 3 \omega C_{N}\right)$

Among them, $C_{N}$ is total grounding capacitance of one-phase line; $R_{N}$ is neutral interposing resistance connects with grounding transformer [1].

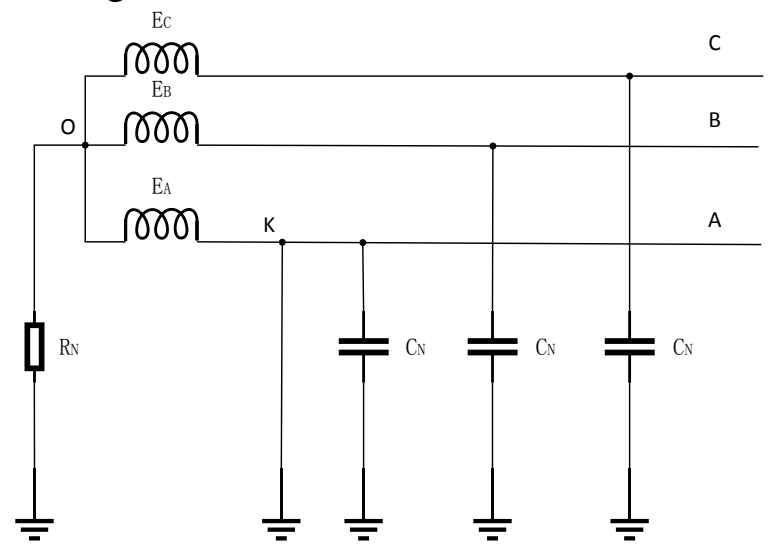

Figure 1 Single-phase equivalent circuit of resistance grounding system 


\subsubsection{Internal overvoltage}

The electric change accumulated by intermittent electric arc can leak into the ground through resistance, while arc grounding over-voltage occurred in resistance grounding system, and neutral potential decreasing rapidly [2]. With the curve of relationship between voltage amplification factor and $I_{R} / I_{C}$, two conclusions can be reached. When $I_{R}>I_{C}$, the sound-phase voltage amplification factor can be controlled under 2.6 times phase voltage; when $I_{R}>1.5 I_{C}$, the effect of overvoltage limit have changed a little. Among them, $I_{R}$ is single-phase grounding neutral resistive current, $I_{C}$ is capacitive current of system [3].

When $I_{R}>1.5 I_{C}$, the condition can be expressed as: $\frac{1}{3 \omega R_{N} C_{N}}>1.5$

\subsubsection{Relay protection}

Based on guaranteeing the reliable action of relay, ground fault current should make the zero-sequence current protection keeps sufficient sensitivity. Therefore, taking the relay protection into consideration, a larger single-phase ground fault current is more favorable. When grounding current is greater than 400-600A, no intermittent arc over-voltage caused by intermittent extinguish, and it is conducive to the right action of relay protection [4]. This paper selects grounding current which is greater than $400 \mathrm{~A}$ that is (take A-phase fault for example) $: E_{A}\left(\frac{1}{R_{N}}+3 \omega C_{N}\right)>400$

\subsubsection{Interference to telecommunication lines}

It has a great influence to telecommunication lines when neutral resistance grounding system occurs single-phase fault. Different regions has different limit to the biggest single-phase fault current. The interference test that $10 \mathrm{kv}$ distribution system to telecommunication lines is conducted in Shanghai, and the result shows that the single-phase ground fault current of $10 \mathrm{kv}$ distribution network is limited to 1000A in Shanghai which can ensure the interference effects within the scope of permit [4]. This paper limits the ground fault current to $1000 \mathrm{~A}$ that is: $E_{A}\left(\frac{1}{R_{N}}+3 \omega C_{N}\right)<1000$

\subsubsection{Personal safety}

When the distribution network ground fault occurs, the main threats for the person near to ground fault spot are contact voltage and step voltage. Multiple actual testing results express that when grounding current in $1000 \mathrm{~A}$, the contact voltage and step voltage in distribution network system are both within the range defined by authority [5]. This paper limits the ground resistance current to 1000 A that is: $\frac{E_{A}}{R_{N}}<1000$

\subsection{Objective function establishment}

When a system failure, the size of neutral grounding resistance can affect the current flowing through the grounding transformer and meanwhile affect the circuital overvoltage. Accordingly, affect the selection of grounding transformer capacity and cable insulation level. Other things being equal, these factors are the major determinant of the price.

This paper selects the price and the minimum to be the objective function that is: $\min F(R)=F_{1}(R)+F_{2}(R)$

Among them, $F(R)$ is the function of the neutral grounding resistance value considered the price of equipment, $F_{l}(R)$ is the function of the neutral grounding resistance value considered the price of grounding transformer, $F_{2}(R)$ is the function of the neutral grounding resistance value considered the price of cable line.

\subsubsection{Relationship between grounding transformer and resistance}

According to the literature [6], short-time capacity of grounding transformer $Q_{d}=U_{L} I_{R} / \sqrt{3}$, rated capacity $Q_{e}=Q_{d} / K$. Among them, $U_{L}$ is power grid rated line voltage, $K$ is overload multiples, refer to the external condition of 10 s thermal stability, take $K$ as 10.5 . The relationship among grounding transformer capacity, price and grounding resistance value as shown in table 1: 
Table 1 Relation table of neutral resistance and transformer price

\begin{tabular}{cccc}
\hline $\begin{array}{c}\text { Grounding } \\
\text { resistance } R_{N} / \Omega\end{array}$ & Neutral current $I_{R} / \mathrm{A}$ & $\begin{array}{c}\text { Transformer } \\
\text { capacity } S_{e} / \text { KVA }\end{array}$ & $\begin{array}{c}\text { Grounding transformer price } \\
\text { (yuan per) }\end{array}$ \\
\hline 350 & 17.32051 & 10 & 6800 \\
70 & 86.60254 & 50 & 15600 \\
35 & 173.2051 & 100 & 21000 \\
17.5 & 346.4102 & 200 & 28000 \\
11.11111 & 545.596 & 315 & 35800 \\
8.75 & 692.8203 & 400 & 41000 \\
7.777778 & 779.4229 & 450 & 45600 \\
7 & 866.0254 & 500 & 48000 \\
6.363636 & 952.6279 & 550 & 49000 \\
5 & 1212.436 & 700 & 56000 \\
4.375 & 1385.641 & 800 & 60000 \\
\hline
\end{tabular}

To fit the data in Figure 1 with curve fitting in MATLAB, a curve between neutral resistance value and grounding transformer price has obtained as shown in Figure 2. The functional relationship is: $F_{1}(R)=12.77 * R_{N}-0.5138$ (ten thousand yuan)

The parameter of fitting degree is: SSE: $0.08486 ; R^{2}: 0.9971$; RMSE: 0.0971 . The result shows that fitting degree can express the relationship between neutral grounding resistance and grounding transformer price correctly.



Figure 2 Relationship between neutral grounding resistance and transformer price

\subsubsection{Insulation selection of cable line}

For $10 \mathrm{kv}$ system, there are two kinds of nominal voltage values of cable: $6 / 10 \mathrm{kv}$ and $8.7 / 10 \mathrm{kv}$ [7], when $U_{t} \leq 21 \mathrm{kv}$, to select the cable line with $6 / 10 \mathrm{kv}$ nominal voltage; when $U_{t}>21 \mathrm{kv}$, to select the cable line with $8.7 / 10 \mathrm{kv}$ nominal voltage.

The size of arc grounding over-voltage will reduce with the decrease of neutral grounding resistance [8]. Therefore, for a certain system, there is always an $R_{\text {mid }}$ makes the arc grounding over-voltage is $21 \mathrm{kv}$, when $R_{N}=\mathrm{R}_{\text {mid. }}$.

Then we can get the function of neutral grounding resistance related with cable line price:

$$
F_{2}(R)=\left\{\begin{array}{l}
X_{1} \times L\left(R_{\text {min }} \leq R_{N} \leq R_{\text {mid }}\right) \\
X_{2} \times L\left(R_{\text {mid }} \leq R_{N} \leq R_{\text {max }}\right)
\end{array}\right\}
$$

Among them, $R_{\min }$ and $R_{\max }$ are the lower limit and upper limit of the value range of $R_{N}$ in constraint condition; $X_{1}$ and $X_{2}$ are the price of $6 / 7 \mathrm{kv}$ cable and $8.7 / 10 \mathrm{kv}$ cable respectively with the same type and same sectional area, and the unit is ten thousand yuan per kilometer; $\mathrm{L}$ is cable length required by system.

\subsection{Decision method}

From the analysis of 2.1 and 2.2, we can get the objective function in this paper:

$\min F(R)=F_{1}(R)+F_{2}(R)=\left\{\begin{array}{l}12.77 \times R_{N}^{-0.5138}+X_{1} \times L\left(R_{\min } \leq R_{N} \leq R_{\text {mid }}\right) \\ 12.77 \times R_{N}^{-0.5138}+X_{2} \times L\left(R_{\text {mid }} \leq R_{N} \leq R_{\max }\right)\end{array}\right.$ 




\section{Conclusion}

The selection of neutral grounding resistance involves every aspects of electrical power system. This paper takes the requirements of internal overvoltage in system, relay protection, interference to telecommunication lines and personal safety as the constraint condition, uses optimal economy as the objective function to establish the optimal selection model of neutral grounding resistance. According to the constraint conditions, the value of neutral grounding resistance is selected as a certain range. In a real case, distribution network has different characteristic in different area, therefore, combining with real situation, we should select the optimum grounding resistance in the reasonable range according to the technical condition and economy.

\section{Acknowledgements}

Thanks for the special fund project (2014QN38) of the fundamental research funds for the central universities.

\section{References}

[1] Gaolong Li. 10kV Distribution Network Single Phase Short-circuit Current Calculation and Step Voltage Finite Element Analysis (Specializing Master, Hunan University, China, 2011). p.34.

[2] Dev Paul and S. I. Venugopalan, Senior Members, IEEE. Low-Resistance Grounding Method for Medium-Voltage Power Systems. Industry F Application Society Annual Meeting, 1991, 2:1571-1578.

[3] Shaoxun Ping, Yufang Zhou. Power system neutral grounding and operation analysis [M] China Electric Power Press, 2010.p.258.

[4] Tiantian Li. Research on Upgrading Neutral Ground Modes and the Relay Protection in 20kV Distribution Network (Master of Engineering Science. Beijing Jiaotong University, China, 2010). p.23.

[5] Qiancheng Ni. Research on Neutral Grounding Mode in 10kV Distribution Network in Hangzhou District (Master of Engineering, North China Electric Power University, China, 2015). p.22.

[6] Xin Wang. Study on Ground Protection for Resistance Grounding Power System (Master of Engineering Science. Shandong University, China, 2014).p.25.

[7] Liang Gao. Power distribution equipment and systems [M]. China Electric Power Press, 2009, p.50.

[8] Guowu Xia, Hong Cui. Research on Power Frequency Arc Grounding Overvoltage of 10kV System [J]. Journal of Northeast Dianli University, Vol.32 (2012), No.6, p.33-37. 